\title{
A Sparse Texture Representation Using Local Affine Regions
}

\author{
Svetlana Lazebnik $^{1} \quad$ Cordelia Schmid $^{2} \quad$ Jean Ponce $^{1}$ \\ slazebni@uiuc.edu cordelia.schmid@inrialpes.fr ponce@cs.uiuc.edu \\ ${ }^{1}$ Beckman Institute \\ ${ }^{2}$ INRIA Rhône-Alpes, \\ University of Illinois \\ 405 N. Mathews Ave. \\ Urbana, IL 61801, USA \\ 665 Avenue de l'Europe, \\ 38330 Montbonnot, France
}

\begin{abstract}
This article introduces a texture representation suitable for recognizing images of textured surfaces under a wide range of transformations, including viewpoint changes and non-rigid deformations. At the feature extraction stage, a sparse set of affine Harris and Laplacian regions is found in the image. Each of these regions can be thought of as a texture element having a characteristic elliptic shape and a distinctive appearance pattern. This pattern is captured in an affine-invariant fashion via a process of shape normalization followed by the computation of two novel descriptors, the spin image and the RIFT descriptor. When affine invariance is not required, the original elliptical shape serves as an additional discriminative feature for texture recognition. The proposed approach is evaluated in retrieval and classification tasks using the entire Brodatz database and a publicly available collection of 1000 photographs of textured surfaces taken from different viewpoints.
\end{abstract}

Keywords: Image Processing and Computer Vision, Feature Measurement, Texture, Pattern Recognition. 


\section{Introduction}

The automated analysis of image textures has been the topic of extensive research in the past forty years, dating back at least to Julesz in 1962 [14]. Existing techniques for modeling texture include co-occurrence statistics [11, 14], filter banks [29, 37], and random fields [30, 51]. Unfortunately, most of these methods make restrictive assumptions about the nature of the input texture (e.g., stationarity), and they are not, in general, invariant with respect to 2D similarity and affine transformations, much less to 3D transformations such as viewpoint changes and non-rigid deformations of the textured surface. Invariance to such transformations is highly desirable for many applications, including wide-baseline stereo matching $[1,40,41,45]$, indexing and retrieval in image and video databases $[32,42,43,44]$, and classification of images of materials $[46,47]$.

In this article, we set out to develop a texture representation that is invariant to geometric transformations that can be locally approximated by an affine model. Since sufficiently small patches on the surfaces of smooth 3D objects are always approximately planar, local affine invariants are appropriate for modeling not only global 2D affine transformations of the image, but also perspective distortions that arise in imaging a planar textured surface, as well as non-rigid deformations that preserve the locally flat structure of the surface, such as the bending of paper or cloth. Specifically, our proposed texture representation is based on characterizing the appearance of distinguished local affine regions in the image. Like other approaches based on textons, or primitive texture elements [7, 22, 46, 47], our method involves representing distributions of 2D image features; unlike these, however, it performs shape selection, ensuring that descriptors are computed over neighborhoods whose shape is adapted to changes in surface orientation and scale caused by viewpoint changes

or scene deformations. In addition, our method relies on spatial selection by computing descriptors at a sparse set of image locations output by local affine region detectors. This is a significant departure from the traditional feature extraction framework, which involves 


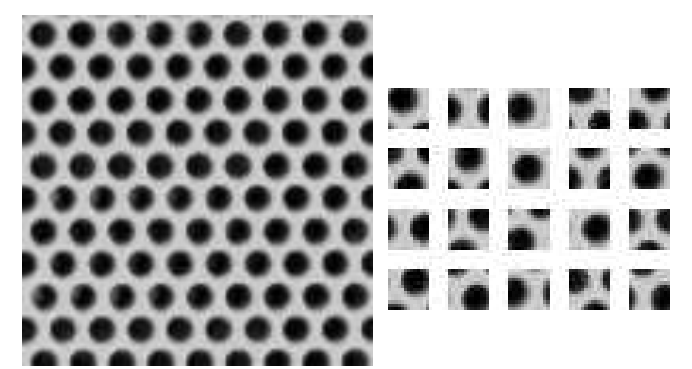

(a) (b)

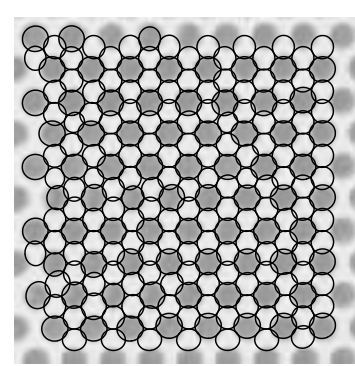

(c)

\section{- EII}

(d)

Figure 1: The effect of spatial selection on a texton dictionary. (a) Original texture image. (b) Top 20 textons found by clustering all $13 \times 13$ patches of the image. (c) A sparse set of regions found by the Laplacian detector described in Section 3.1. Each region is normalized to yield a $13 \times 13$ patch. (d) Textons obtained by clustering the normalized patches. ${ }^{1}$

processing every pixel location in the image. Apart from being memory- and computationintensive, this dense approach produces redundant texton dictionaries that may include, for instance, many slightly shifted versions of the same basic element [28]. As Figure 1 illustrates, spatial selection is an effective way to reduce this redundancy.

Our approach consists of the following steps (see also Figure 2):

1. Extract a sparse set of affine regions ${ }^{2}$ in the shape of ellipses from a texture image. The two region detectors used for this purpose are described in Section 3.1.

2. Normalize the shape of each elliptical region by transforming it into a circle. This reduces the affine ambiguity to a rotational one. Full affine invariance is achieved by computing rotation-invariant descriptors over the normalized regions. In Section 3.2, we introduce two novel rotation-invariant descriptors: one based on spin images used for matching range data [13], and one based on Lowe's SIFT descriptor [27].

3. Perform clustering on the affine-invariant descriptors to obtain a more compact repre-

\footnotetext{
${ }^{1}$ For the sake of this illustration, we disregard the orthogonal ambiguity inherent in the normalization process (see Section 3.1 for details). Because we are clustering the normalized patches themselves, instead of rotation-invariant descriptors as in Section 3.2, the resulting description of patch appearance in this case is rotation-dependent. This can be seen from the fact that the second and third clusters of (d) are rotated versions of each other.

${ }^{2}$ More accurately, they should be termed affine-covariant: In principle, the regions found in a picture deformed by some affine transformation are the images of the regions found in the original picture under the same transformation.
} 
sentation of the distribution of features in each image (Section 3.3). Summarize this distribution in the form of a signature, containing a representative descriptor from each cluster and a weight indicating the relative size of the cluster.

4. Compare signatures of different images using the Earth Mover's Distance (EMD) [38, 39], which is a convenient and effective dissimilarity measure applicable to many types of image information. The output of this stage is an EMD matrix whose entries record the distances between each pair of signatures in the database. The EMD matrix can be used for retrieval and classification tasks, as described in Section 4.1.

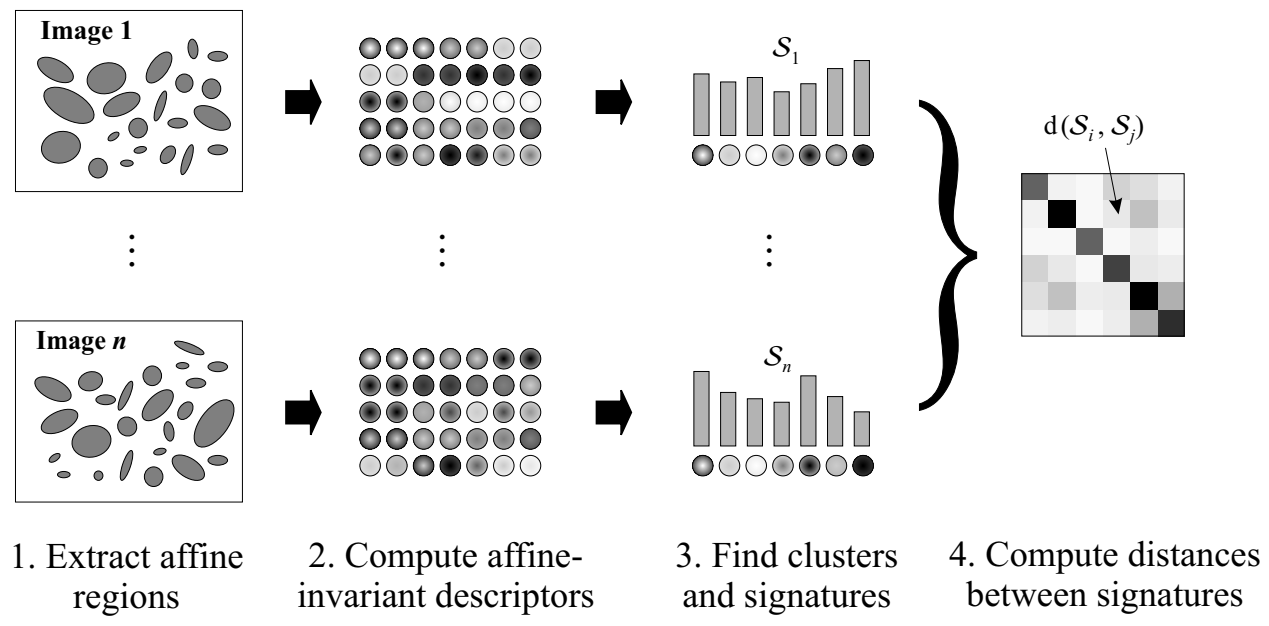

Figure 2: The architecture of the feature extraction system proposed in this article.

In Section 4, we will use two datasets to evaluate the capabilities of the proposed texture representation. The first dataset, introduced in Section 4.2, consists of photographs of textured surfaces taken from different viewpoints and featuring large scale changes, perspective distortions, and non-rigid transformations. Even though our method relies on implicit assumptions of local flatness and Lambertian appearance, and is thus theoretically applicable primarily to "albedo textures" due to spatial albedo variations on smooth surfaces, the results in Section 4.2 show that in practice it also tends to perform well on "3D textures" arising from local relief variations on a surface. Our second set of experiments, described in Section 4.3, is carried out on the Brodatz database [4], a collection of images that features significant inter-class variability, but no geometric transformations between members of the 
same class. Because affine invariance is not required in this case, we modify the basic feature extraction framework to use neighborhood shape as a discriminative feature to improve performance.

\section{Previous Work}

Before presenting the details of our approach (Section 3), let us briefly discuss a number of texture models aimed at achieving invariance under various geometric transformations. Early research in this domain has concentrated on global 2D image transformations, such as rotation and scaling $[5,30]$. However, such models do not accurately capture the effects of 3D transformations (even in-plane rotations) of textured surfaces. More recently, there has been a great deal of interest in recognizing images of textured surfaces subjected to lighting and viewpoint changes $[7,8,22,26,46,47,49]$. A few methods $[22,26,49]$ are based on explicit reasoning about the $3 \mathrm{D}$ structure of the surface, which may involve registering samples of a material so that the same pixel across different images corresponds to the same physical point on the surface [22] or applying photometric stereo to reconstruct the depth map of the 3D texture $[26,49]$. While such approaches capture the appearance variations of 3D surfaces in a principled manner, they require specially calibrated datasets collected under controlled laboratory conditions. For example, the 3D texton representation of Leung and Malik [22] naturally lends itself to the task of classifying a "stack" of registered images of a test material with known imaging parameters, but its applicability is limited in most practical situations.

In this presentation, we are interested in classifying unregistered texture images. This problem has been addressed by Cula and Dana [7] and Varma and Zisserman [46, 47], who have developed several dense 2D texton-based representations capable of very high accuracy on the challenging Columbia-Utrecht reflectance and texture (CUReT) database [8]. The descriptors used in these representations are filter bank outputs [7, 46] and raw pixel values [47]. Even though these methods have been successful in the complex task of classifying images of materials despite significant appearance changes, the representations themselves 


\begin{tabular}{|l|l|l|}
\hline Components & Previous work $[7,22,46,47]$ & This article \\
\hline Spatial selection & None: every pixel is considered & $\begin{array}{l}\text { Laplacian and Harris affine region } \\
\text { detectors [9, 33] }\end{array}$ \\
\hline Neighborhood shape selection & None: neighborhood size is fixed & Affine adaptation process [9] \\
\hline Descriptor computation & $\begin{array}{l}\text { Filter banks }[7,22,46], \text { pixel val- } \\
\text { ues [47] }\end{array}$ & $\begin{array}{l}\text { Novel descriptors: spin images, } \\
\text { RIFT }\end{array}$ \\
\hline Finding textons & $\begin{array}{l}\text { Clustering, universal texton dic- } \\
\text { tionaries }\end{array}$ & $\begin{array}{l}\text { Clustering, separate texton rep- } \\
\text { resentation for each image }\end{array}$ \\
\hline $\begin{array}{l}\text { Representing/comparing texton } \\
\text { distributions }\end{array}$ & Histograms $/ \chi^{2}$ distance & $\begin{array}{l}\text { Signatures/Earth Mover's Dis- } \\
\text { tance [39, 38] }\end{array}$ \\
\hline
\end{tabular}

Table 1: The components of our approach, contrasted with other 2D texton-based methods. are not invariant to the changes in question. In particular, the support regions for computing descriptors are the same in all images; no adaptation is performed to compensate for changes in surface orientation with respect to the camera.

Because they lack representation-level invariance, the above methods require the use of multiple models or prototypes to represent a single texture. As long as the training set adequately samples all viewpoints and lighting directions that can be encountered at test time, the texture can be successfully recognized. On the other hand, when the test set contains images not represented in the training set (e.g., images at significantly different scales), performance drops dramatically [46]. In our work, we shift away from this dependence on representative training sets by developing a texture representation with built-in geometric invariance. Note, however, that our present method does not explicitly account for changes in lighting direction and associated 3D effects such as self-shadowing; therefore, to achieve robust recognition in the presence of such effects, we must still rely on multiple prototypes in the training set.

Table 1 summarizes the main components of our approach and contrasts it with other 2D texton-based methods. A preliminary version of this article has appeared in [21]. 


\section{Components of the Representation}

\subsection{Affine Regions}

Conceptually, our approach may be traced back to early articles on the extraction of local features in natural images, where emphasis is placed on locating perceptually salient primitives such as blobs [6, 48]. Blostein and Ahuja [3] were the first to introduce a multiscale blob detector based on maxima of the Laplacian. Lindeberg [24] has extended this detector in the framework of automatic scale selection, where a "blob" is defined by a scale-space location where a normalized Laplacian measure attains a local maximum. Informally, the spatial coordinates of the maximum become the coordinates of the center of the blob, and the scale at which the maximum is achieved becomes its characteristic scale. Gårding and Lindeberg [9] have also shown how to design an affine blob detector using an affine adaptation process based on the second moment matrix. This process forms an important part of the affine-invariant region detection frameworks of Baumberg [1] and Mikolajczyk and Schmid [33]. Both of these methods rely on a multiscale version of the Harris operator [12] to localize interest points in space. Alternative region extraction schemes include the "entropy detector" of Kadir and Brady [16], the difference-of-Gaussians (or DoG) detector of Lowe [27], the "maximally stable extremal regions" of Matas et al. [31], and the cornerand intensity-based operators of Tuytelaars and Van Gool [45]. Of the above, [16, 27] are scale-invariant, while [31, 45] are fully affine-invariant. This proliferation of region detectors, motivated primarily by applications to wide-baseline stereo matching and image retrieval, attests to the increased importance accorded to the spatial and shape selection principles in the computer vision community.

In this work, we use two types of detectors: the Harris-affine detector of Mikolajczyk and Schmid [33] and the Laplacian blob detector of Gårding and Lindeberg [9]. ${ }^{3}$ Figure 3 shows

\footnotetext{
${ }^{3}$ Note that we have implemented simplified versions of the algorithms given in [9, 33]. In particular, the affine adaptation process is not iterated, and the local (differentiation) scale is fixed, instead of being automatically determined at each iteration. See also [1, 40, 41] for related approaches to region extraction.
} 

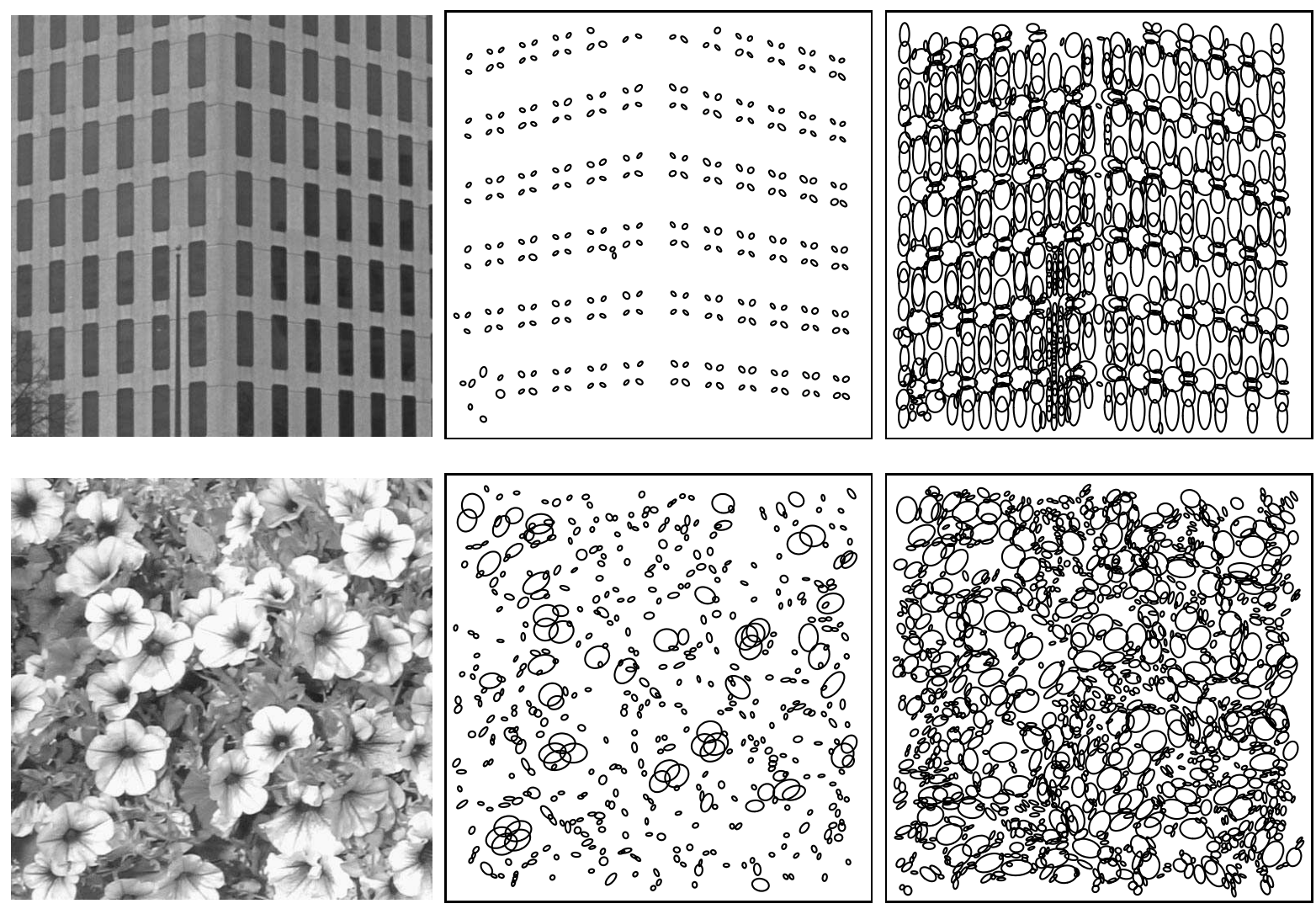

Figure 3: Output of the Harris and Laplacian region detectors on two natural images. Left: original images, center: regions found by the Harris detector, right: regions found by the Laplacian detector. Note that the Laplacian detector tends to produce a denser set of regions than the Harris.

the output of the detectors on two sample images. Note that the Harris detector tends to find corners and points at which significant intensity changes occur, while the Laplacian detector is (in general) attracted to points that can be thought of as centers of roughly elliptical regions of uniform intensity. Intuitively, the two detectors provide complementary kinds of information about the image: The former responds to regions of "high information content" [33], while the latter produces a perceptually plausible decomposition of the image into a set of blob-like primitives.

The technical details of automatic scale selection and affine adaptation, described in $[9$, $24,33]$, are beyond the scope of this article. For our purposes, it is sufficient to note that the affinely adapted regions localized by the Harris and Laplacian detectors are represented as 
ellipses. We can normalize these regions by mapping the corresponding ellipses onto a unit circle. Because the circle is invariant under rotations and reflections, it can be easily shown that the normalization process has an inherent orthogonal ambiguity. In some previous work, this ambiguity has been resolved by estimating a dominant gradient direction of the patch and aligning this direction with the positive x-axis [27, 33]. However, in our experience the dominant orientation estimates have tended to be unreliable, especially for Laplacian regions, which lack strong edges at the center. To avoid the potential instability and the computational expense of finding the dominant gradient, we have chosen instead to represent each normalized patch by a rotationally invariant descriptor. A similar strategy has been followed by $[1,41]$.

Note. To achieve invariance to local affine transformations, as in the experiments of Section 4.2, we discard the information contained in the affine shape of the patches. However, as a glance at Figure 3 suggests, this shape can be a distinctive feature when affine invariance is not required. This point will be revisited in Section 4.3 .

\subsection{Rotation-Invariant Descriptors}

In this article, we introduce two novel rotation-invariant descriptors: intensity-domain spin images, inspired by the method for matching range data developed by Johnson and Hebert [13]; and RIFT descriptors, based on the Scale-Invariant Feature Transform (SIFT) developed by Lowe [27]. We will conclude this section by discussing the advantages of the proposed descriptors over the more traditional features like differential invariants [18, 43] and filter banks $[1,7,41,42,46]$.

Intensity-domain spin images. Our first rotation-invariant descriptor is inspired by the spin images introduced by Johnson and Hebert [13] for matching range data. The intensitydomain spin image proposed in this article is a two-dimensional histogram encoding the distribution of image brightness values in the neighborhood of a particular reference (center) point. The two dimensions of the histogram are $d$, distance from the center point, and 
$i$, the intensity value. The "slice" of the spin image corresponding to a fixed $d$ is simply the histogram of the intensity values of pixels located at a distance $d$ from the center. Since the $d$ and $i$ parameters are invariant under orthogonal transformations of the image neighborhood, spin images offer an appropriate degree of invariance for representing affinenormalized patches. In the experiments reported in Section 4, we used 10 bins for distance and 10 for intensity value, resulting in 100-dimensional descriptors.

We implement the spin image as a "soft histogram" where each pixel within the support region contributes to more than one bin. Specifically, the contribution of a pixel located in $x$ to the bin indexed by $(d, i)$ is given by

$$
\exp \left(-\frac{\left(\left|x-x_{0}\right|-d\right)^{2}}{2 \alpha^{2}}-\frac{|I(x)-i|^{2}}{2 \beta^{2}}\right),
$$

where $x_{0}$ is the location of the center pixel, and $\alpha$ and $\beta$ are the parameters representing the "soft width" of the two-dimensional histogram bin. Note that the soft histogram can be seen as a set of samples from the Parzen estimate (with Gaussian windows) of the joint density of intensity values $i$ and distances $d$. The use of soft histograms has also been advocated by Koenderink and Van Doorn [19] because it alleviates aliasing effects. Figure 4 shows the principle behind the construction of spin images.

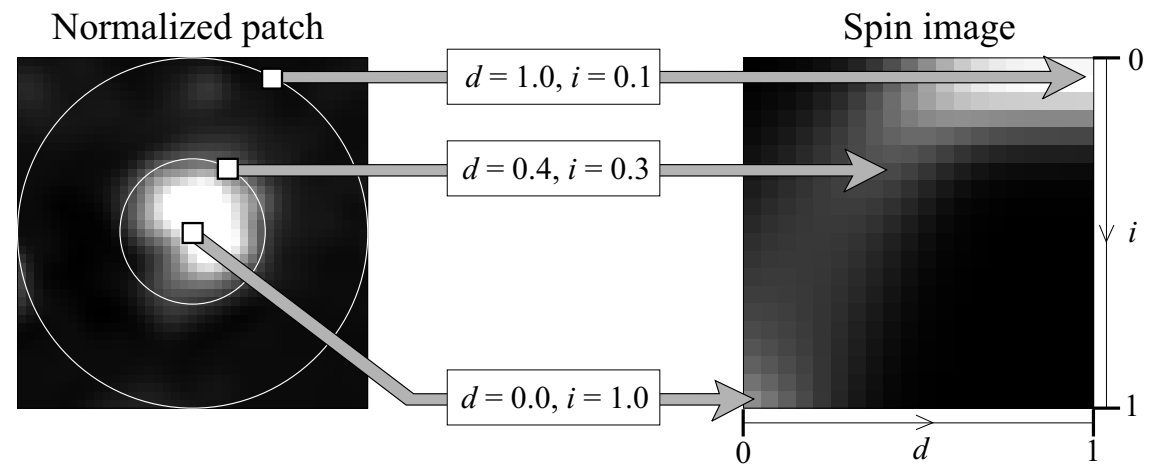

Figure 4: Construction of spin images. Three sample points in the normalized patch (left) map to three different locations in the descriptor (right).

To achieve invariance to affine transformations of the image intensity function (that is, transformations of the form $I \mapsto a I+b)$, it is sufficient to normalize the range of the intensity function within the support region of the spin image [40]. To alleviate the potential 
sensitivity of the normalization to noise and resampling artifacts (these are particularly severe for patches that are only a few pixels wide), we slightly blur the normalized patches with a Gaussian kernel before computing the spin image.

RIFT descriptors. To obtain a complementary representation of local appearance of normalized patches, we have developed an additional rotation-invariant descriptor that generalizes Lowe's SIFT [27]. The original SIFT has been noted for its superior performance in retrieval tasks [34]; however, we cannot use it directly in our work because it depends on finding the dominant orientation of the normalized patch. Our descriptor, dubbed RotationInvariant Feature Transform, or RIFT, is constructed as follows. The circular normalized patch is divided into concentric rings of equal width, and a gradient orientation histogram is computed within each ring (Figure 5). To maintain rotation invariance, this orientation is measured at each point relative to the direction pointing outward from the center. We use four rings and eight histogram orientations, yielding 32-dimensional descriptors. ${ }^{4}$ Note that the RIFT descriptor as described above is not invariant to flipping of the normalized patch, which reverses the order of directions in the orientation histogram. However, we are not concerned with this circumstance in our current work, since realistic imaging conditions do not involve reversing the orientation of a textured surface.
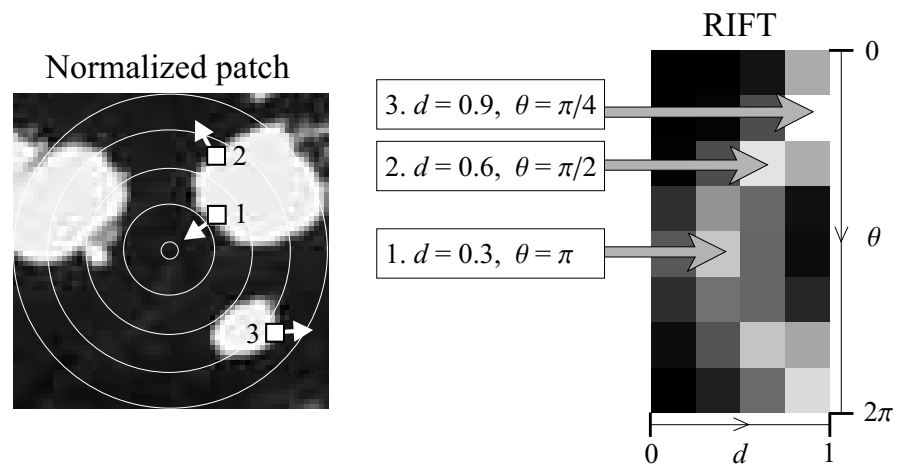

Figure 5: Construction of RIFT descriptors. Three sample points in the normalized patch (left) map to three different locations in the descriptor (right).

\footnotetext{
${ }^{4}$ The original SIFT descriptor has 128 dimensions, as it is based on subdividing a square image patch into 16 smaller squares. To achieve rotation invariance, we must use concentric rings instead, hence the drop in dimensionality for RIFT.
} 
Discussion. Both spin images and RIFT descriptors follow the same strategy as the original SIFT and other recently developed features like shape contexts [2]: They subdivide the region of support and compute a histogram of appearance attributes (most frequently, pixel values or gradient orientations) inside each subregion. Histogramming provides stability against deformations of the image pattern, while subdividing the support region offsets the potential loss of spatial information. In this way, a compromise is achieved between the conflicting requirements of greater geometric invariance on the one hand and greater discriminative power on the other. Intuitively, it seems plausible that descriptors based on this compromise would be simultaneously richer and more robust than traditional descriptors that compute functions of the entire region of support, namely, filter banks and differential invariants. Recent experimental data tends to support this intuition: SIFT descriptors have achieved better repeatability rates than filter banks and differential invariants in the comparative evaluations of Mikolajczyk and Schmid [34], while shape contexts have performed remarkably well for the application of handwritten digit recognition [2]. In addition, at an earlier stage of this research [21], spin images have achieved better results than rotation-invariant "Gabor-like" linear filters [42] (note that this filter bank is similar in terms of performance and dimensionality to several others in popular use [46], and superior to differential invariants [42]).

In this article, we perform a comparative evaluation of spin images and RIFT. As will be seen from Section 4, spin images tend to perform better (possibly due to their higher dimensionality). However, combining the two descriptors in a unified recognition framework generally produces better results than using either one in isolation. This may be a reflection of the fact that spin images and RIFT rely on complementary kinds of image information — the former uses normalized graylevel values, while the latter uses the gradient. 


\subsection{Signatures and the Earth Mover's Distance}

One commonly thinks of a texture image as being "generated" by a few basic primitives, or textons [15], repeated many times and arranged in some regular or stochastic spatial pattern. In the field of texture analysis, clustering is the standard technique for discovering a small set of primitives based on a large initial collection of texture element instances. Accordingly, we perform clustering on each texture image separately to form its signature $\left\{\left(m_{1}, u_{1}\right),\left(m_{2}, u_{2}\right), \ldots,\left(m_{k}, u_{k}\right)\right\}$, where $k$ is the number of clusters, $m_{i}$ is the center of the $i$ th cluster, and $u_{i}$ is the relative weight of the cluster (in our case, the size of the cluster divided by the total number of descriptors extracted from the image). Signatures have been introduced by Rubner et al. [38, 39] as representations suitable for matching using the Earth Mover's Distance (EMD). The EMD between two signatures $\mathcal{S}_{1}=\left\{\left(m_{1}, u_{1}\right),\left(m_{2}, u_{2}\right), \ldots,\left(m_{k}, u_{k}\right)\right\}$ and $\mathcal{S}_{2}=\left\{\left(n_{1}, v_{1}\right),\left(n_{2}, v_{2}\right), \ldots,\left(n_{l}, v_{l}\right)\right\}$ has the form

$$
\mathrm{d}\left(\mathcal{S}_{1}, \mathcal{S}_{2}\right)=\frac{\sum_{i} \sum_{j} f_{i j} \mathrm{~d}\left(m_{i}, n_{j}\right)}{\sum_{i} \sum_{j} f_{i j}},
$$

where the scalars $f_{i j}$ are flow values that are determined by solving a linear programming problem, and the scalars $\mathrm{d}\left(m_{i}, n_{j}\right)$ are the ground distances between different cluster centers. The theoretical justification of this formula and the specifics of the optimization setup are beyond the scope of this paper; we refer the interested reader to $[23,39]$ for more details. In our case, $m_{i}$ and $n_{j}$ may be spin images and RIFT descriptors, and the ground distance is simply the Euclidean distance. Since our descriptors are normalized to have unit norm, the ground distances lie in the range $[0,2]$. We rescale this range to $[0,1]$, thus ensuring that all EMD's are between 0 and 1 as well.

For our application, the signature/EMD framework offers several advantages over the alternative histogram $/ \chi^{2}$ distance framework $[7,22,46,47]$. A signature is more robust and descriptive than a histogram, and it avoids the quantization and binning problems associated with histograms, especially in high dimensions [39] (recall that our spin images and RIFT descriptors are 100- and 32-dimensional, respectively). The EMD has been shown to be 
(relatively) insensitive to the number of clusters, i.e., when one of the clusters is split during signature computation, replacing a single center with two, the resulting EMD matrix is not much affected [38]. This is a very important property, since automatic selection of the number of clusters remains an unsolved problem. In addition, in several evaluations of colorand texture-based image retrieval [36, 39], EMD has performed better than other methods for comparing distributions, including $\chi^{2}$ distance. Finally, the EMD/signature framework has the advantage of efficiency and modularity: It frees us from the necessity of clustering descriptors from all images together and computing a universal texton dictionary, which may not represent all texture classes equally well [7].

\section{Experimental Evaluation}

\subsection{Evaluation Strategy}

Channels. Tuytelaars and Van Gool [45] have articulated the goal of building an opportunistic neighborhood extraction system that would combine the output of several region detectors tuned to different kinds of image structure. In this spirit, the texture representation proposed in this article is designed to support multiple region detectors and descriptors. Each detector/descriptor pair is treated as an independent channel that generates its own signature representation for each image in the database, and its own EMD matrix of pairwise inter-image distances. To combine the outputs of several channels, we simply add the corresponding entries in the EMD matrices. This approach was empirically determined to be superior to forming linear combinations with varying weights, or taking the minimum or maximum of the distances.

Since our experimental setup involves the evaluation of two region detectors and two descriptors, we end up with four channels: Harris regions and spin images (HS), Harris regions and RIFT descriptors (HR), Laplacian regions and spin images (LS), and finally, Laplacian regions and RIFT descriptors (LR). In addition, we will introduce in Section 4.3 the Harris and Laplacian ellipse channels, denoted HE and LE, respectively. To simplify the 
notation for combined channels, we will use (in a purely formal, "syntactic" manner) the distributive law: For example, we will write $(\mathrm{H}+\mathrm{L}) \mathrm{R}$ instead of $\mathrm{HR}+\mathrm{LR}$ for the combination of the Harris/RIFT and Laplacian/RIFT channels, and $(\mathrm{H}+\mathrm{L})(\mathrm{S}+\mathrm{R})$ for the combination of all four detector/descriptor channels.

Retrieval. We use the standard procedure followed by several Brodatz database evaluations $[25,35,50]$. Given a query image, we select other images from our database in increasing order of EMD, i.e., from the most similar to the least similar. Each image in the database is used as a query image once, and the performance is summarized as a plot of average recall vs. the number of retrievals. Average recall is defined as the number of images retrieved from the same class as the query image over the total number of images in that class (minus one to account for the query itself), averaged over all the queries. For example, perfect performance for a given class would correspond to average recall of $100 \%$ after $n-1$ retrievals, where $n$ is the number of images in that class.

Classification. In effect, the evaluation framework described above measures how well each texture class can be modeled by individual samples. It is not surprising that retrieval can fail in the presence of sources of variability that are not fully accounted for by the invariance properties of the representation (recall that our representation provides invariance to local geometric deformations and affine illumination changes, but not to complex viewpoint- and lighting-dependent appearance changes). To obtain a more balanced assessment of performance, a texture representation should be evaluated using classification as well as retrieval. In the classification framework, a model for a class is created not from a single (possibly atypical) image, but from a set of multiple training images, thereby compensating for the effects of intra-class variability.

In our implementation, we use nearest-neighbor classification with EMD. The training set is selected as a fixed-size random subset of the class, and all remaining images comprise the test set. To eliminate the dependence of the results on the particular training images used, we report the average of the classification rates obtained for different randomly selected 
training sets. More specifically, a single sequence of 200 random subsets is generated and used to evaluate all the channel combinations seen in Tables 2 and 4. This ensures that all the rates are directly comparable, i.e., small differences in performance cannot be attributed to random "jitter".

\subsection{Dataset 1: Textured Surfaces}

To test the invariance properties of our proposed representation, we have collected a texture database consisting of 1000 uncalibrated, unregistered images: 40 samples each of 25 different textures. The database is publicly available at http://www-cvr.ai.uiuc.edu/ponce_grp. Figure 6 shows four sample images from each class (the resolution of the samples is $640 \times 480$ pixels). The database includes surfaces whose texture is due mainly to albedo variations (e.g., wood and marble), 3D shape (e.g., gravel and fur), as well as a mixture of both (e.g., carpet and brick). Significant viewpoint changes and scale differences are present within each class, and illumination conditions are uncontrolled. During data acquisition, we have taken care to exercise additional sources of variability wherever possible. These include non-planarity of the textured surface (bark), significant non-rigid deformations between different samples of the same class (fur, fabric, and water), inhomogeneities of the texture pattern (bark, wood, and marble), and viewpoint-dependent appearance variations (glass).

Each image in the database is processed with the Harris and Laplacian detectors. The median number of Harris (resp. Laplacian) regions extracted per image is 926 (resp. 4591). The median number of combined regions is 5553, or about $1.8 \%$ of the total number of pixel locations in the image. Thus, we can see that the spatial selection performed by the detectors results in a drastic compression of the amount of data that needs to be handled by the subsequent processing stages, especially clustering, which is a notoriously memoryintensive operation. In our implementation, clustering was performed using the $k$-means algorithm with $k=40$ centers. 


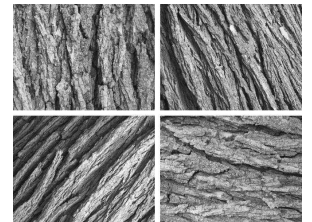

T01 (bark)

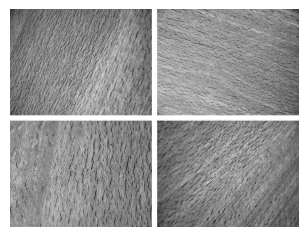

T06 (wood)

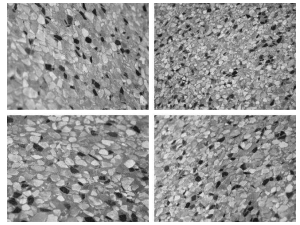

T11 (stone)

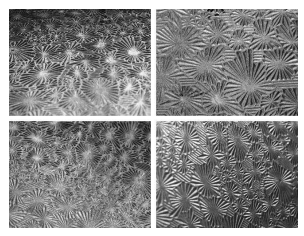

T16 (glass)

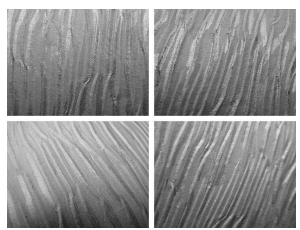

T21 (paper)

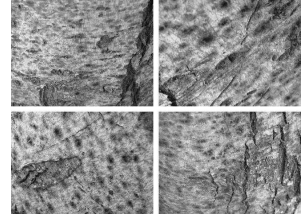

T02 (bark)

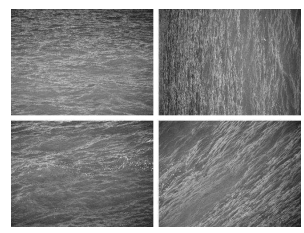

T07 (water)

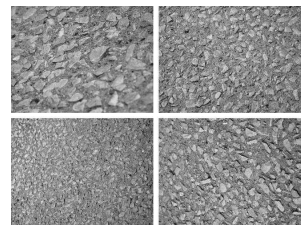

T12 (gravel)

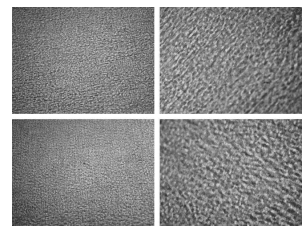

T17 (glass)

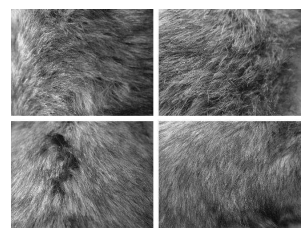

T22 (fur)

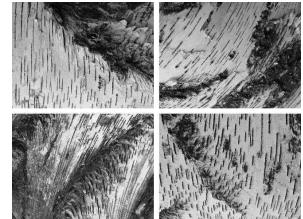

T03 (bark)

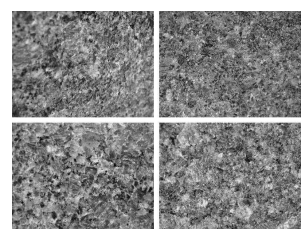

T08 (granite)

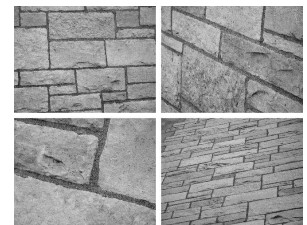

T13 (wall)

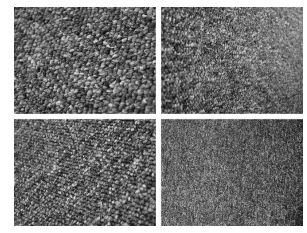

T18 (carpet)

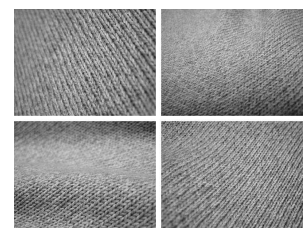

T23 (fabric)

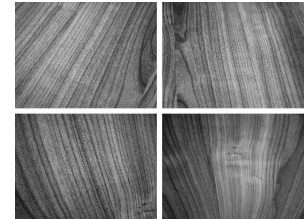

T04 (wood)

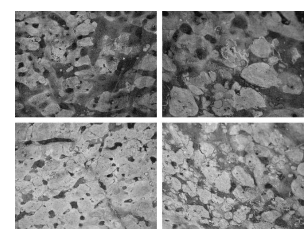

T09 (marble)

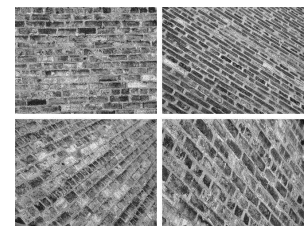

T14 (brick)

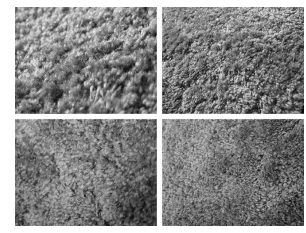

T19 (carpet)

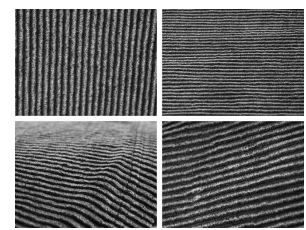

T24 (fabric)

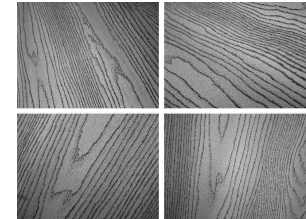

T05 (wood)

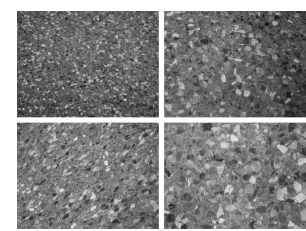

T10 (stone)

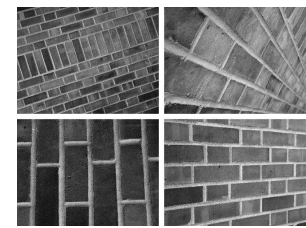

T15 (brick)

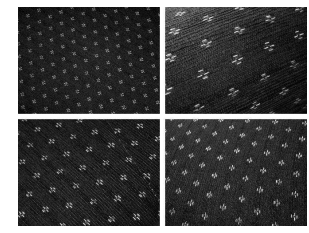

T20 (fabric)

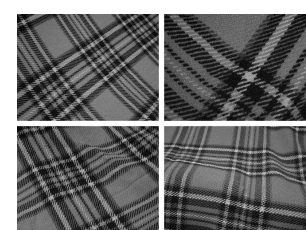

T25 (fabric)

Figure 6: Four samples each of the 25 texture classes used in the experiments of Section 4.2. The entire database may be downloaded from http://www-cvr.ai.uiuc.edu/ponce_grp. 


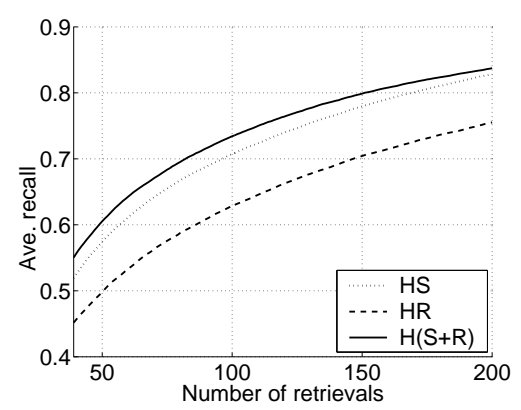

(a)

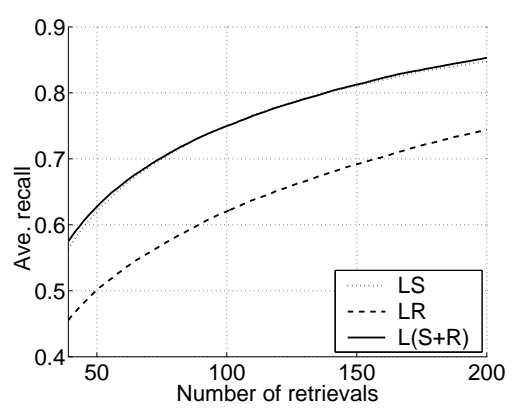

(b)

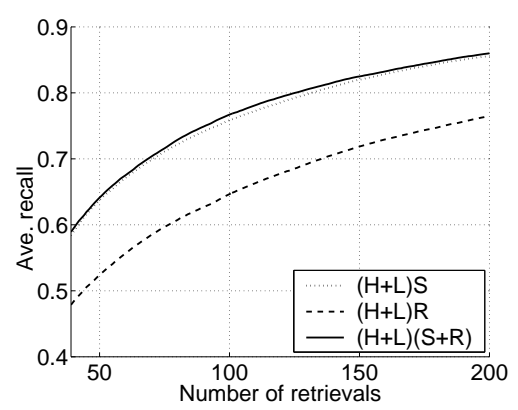

(c)

Figure 7: Retrieval curves for the texture database. (a) The Harris channels. (b) The Laplacian channels. (c) The combined Harris and Laplacian channels.

Figure 7 shows retrieval results for the texture database. The first observation is that spin images perform better than RIFT descriptors, and the combination of the two descriptors performs slightly better than spin images alone. Next, Laplacian regions (part (b) of the figure) perform better than Harris (a), and the combination of the two (c) is slightly better than Laplacian alone. The solid curve in part (c) of the figure shows the retrieval performance obtained by combining all four detector/descriptor channels. The recall after 39 retrievals is $58.92 \%$. This relatively low number is a reflection of the considerable intra-class variability of the database. As discussed in Section 4.1, we cannot expect that all samples of the same class will be well represented by a single prototype. Accordingly, the combined $(\mathrm{H}+\mathrm{L})(\mathrm{S}+\mathrm{R})$ classification rate is only $62.15 \%$ for one training sample, but it goes up rapidly to $92.61 \%$ for 10 samples and $96.03 \%$ for 20 samples. Table 2 shows a comparison of 10 -sample classification rates for different channel combinations. The same trends that were seen in Figure 7 are echoed here: Spin images perform better than RIFT, Laplacian regions perform better than Harris, and the combination $(\mathrm{H}+\mathrm{L})(\mathrm{S}+\mathrm{R})$ has the highest performance.

We may wonder whether the superior performance of Laplacian points is due to the denser representation they afford (recall that the Laplacian detector finds almost five times as many regions as the Harris). To check this conjecture, we have repeated the recognition experiments after thresholding the output of the Laplacian detector so that equal numbers of Laplacian and Harris regions are produced for each image. The results of the "truncated" 


\begin{tabular}{|l|l|l|l|}
\hline & $\mathrm{H}$ & $\mathrm{L}$ & $\mathrm{H}+\mathrm{L}$ \\
\hline $\mathrm{S}$ & 0.8332 & 0.8829 & 0.9015 \\
\hline $\mathrm{R}$ & 0.7927 & 0.8318 & 0.8547 \\
\hline $\mathrm{S}+\mathrm{R}$ & 0.8814 & 0.9196 & 0.9261 \\
\hline
\end{tabular}

Table 2: Classification results for 10 training samples per class. First column (top to bottom): HS, HR, H(S+R). Second column: LS, LR, L $(\mathrm{S}+\mathrm{R})$. Third column: $(\mathrm{H}+\mathrm{L}) \mathrm{S},(\mathrm{H}+\mathrm{L}) \mathrm{R}$, $(\mathrm{H}+\mathrm{L})(\mathrm{S}+\mathrm{R})$.

and "full" Laplacian representations may be compared by looking at columns 3 and 4 of Table 3. Interestingly, while the rates may vary significantly for individual textures, the averages (bottom row) are almost the same: $91.93 \%$ and $91.96 \%$ for "truncated" and "full", respectively. Thus, recognition performance cannot be regarded as a simple function of the density of the representation.

Finally, Table 3 allows us to analyze the "difficulty" of individual textures for our system. To this end, the textures are arranged in order of increasing $(\mathrm{H}+\mathrm{L})(\mathrm{S}+\mathrm{R})$ classification rate (last column). Roughly speaking, classification rate is positively correlated with the homogeneity of the texture: Some of the lowest rates belong to inhomogeneous coarse-scale textures like bark (T02, T03) and marble (T09), while some of the highest belong to homogeneous fine-scale textures like glass (T17), water (T07), and fabric (T20, T24). However, this relationship is not universal. For example, granite (T08), which is fine-grained and quite uniform, has a relatively low rate of $86.78 \%$, while the large-scale, inhomogeneous wall (T13) has a relatively high rate of $95.92 \%$. It is also interesting (and somewhat unexpected) that the performance of different classes does not depend on their nature as 3D or albedo textures. Overall, the intrinsic characteristics of the various textures do not seem to provide a clear pattern for predicting performance. This is not surprising if one keeps in mind that classification performance is not related directly to intra-class variability, but to the extent of separation between the classes in feature space. 


\begin{tabular}{|c|c|c|c|c|c|}
\hline & Class & $\mathrm{H}(\mathrm{S}+\mathrm{R})$ & $\mathrm{L}(\mathrm{S}+\mathrm{R})$ (trunc.) & $\mathrm{L}(\mathrm{S}+\mathrm{R})($ full $)$ & $(\mathrm{H}+\mathrm{L})(\mathrm{S}+\mathrm{R})$ \\
\hline & T03 (bark) & 0.7455 & 0.7248 & 0.7512 & 0.7953 \\
\hline & T19 (carpet) & 0.7270 & 0.8592 & 0.8207 & 0.8107 \\
\hline & T02 (bark) & 0.8077 & 0.8167 & 0.8018 & 0.8467 \\
\hline & T08 (granite) & 0.8352 & 0.8855 & 0.8808 & 0.8678 \\
\hline & T09 (marble) & 0.7515 & 0.8360 & 0.8950 & 0.8773 \\
\hline & T21 (paper) & 0.7445 & 0.9335 & 0.9230 & 0.8880 \\
\hline & T16 (glass) & 0.7610 & 0.9753 & 0.9367 & 0.8882 \\
\hline & T12 (gravel) & 0.7947 & 0.8703 & 0.9073 & 0.9012 \\
\hline & T14 (brick) & 0.8307 & 0.9048 & 0.8965 & 0.9028 \\
\hline & T01 (bark) & 0.8972 & 0.9295 & 0.8703 & 0.9063 \\
\hline & T23 (fabric) & 0.8898 & 0.9512 & 0.9082 & 0.9198 \\
\hline & T15 (brick) & 0.8987 & 0.9035 & 0.9067 & 0.9238 \\
\hline & T11 (stone) & 0.8920 & 0.9448 & 0.9488 & 0.9372 \\
\hline & T05 (wood) & 0.8983 & 0.8163 & 0.9250 & 0.9442 \\
\hline & T10 (stone) & 0.8500 & 0.9488 & 0.9687 & 0.9492 \\
\hline & $\mathrm{T} 22$ (fur) & 0.9453 & 0.9423 & 0.9208 & 0.9508 \\
\hline & T25 (fabric) & 0.9330 & 0.9590 & 0.9343 & 0.9590 \\
\hline & T13 (wall) & 0.9288 & 0.9508 & 0.9590 & 0.9592 \\
\hline & T06 (wood) & 0.9690 & 0.9535 & 0.9290 & 0.9743 \\
\hline & T18 (carpet) & 0.9660 & 0.9282 & 0.9800 & 0.9747 \\
\hline & T04 (wood) & 0.9868 & 0.9862 & 0.9618 & 0.9853 \\
\hline & T20 (fabric) & 0.9908 & 0.9702 & 0.9973 & 0.9948 \\
\hline & T07 (water) & 0.9980 & 0.9940 & 0.9905 & 0.9975 \\
\hline & T24 (fabric) & 0.9937 & 0.9990 & 0.9780 & 0.9980 \\
\hline & T17 (glass) & 1.0000 & 1.0000 & 0.9985 & 1.0000 \\
\hline & Mean & 0.8814 & 0.9193 & 0.9196 & 0.9261 \\
\hline
\end{tabular}

Table 3: Detailed breakdown of classification results summarized in Table 2. The classes are sorted in order of increasing classification rate (last column). See text for discussion. 


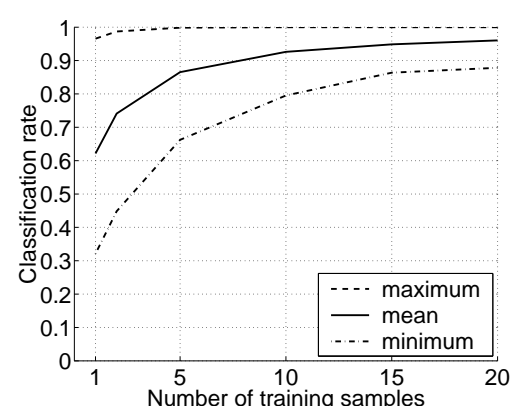

(a) $(\mathrm{H}+\mathrm{L})(\mathrm{S}+\mathrm{R})$

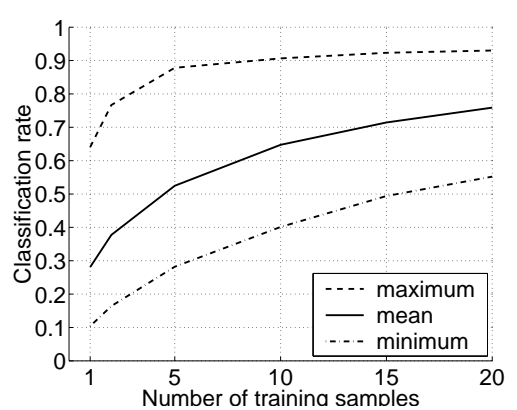

(b) VZ-Joint

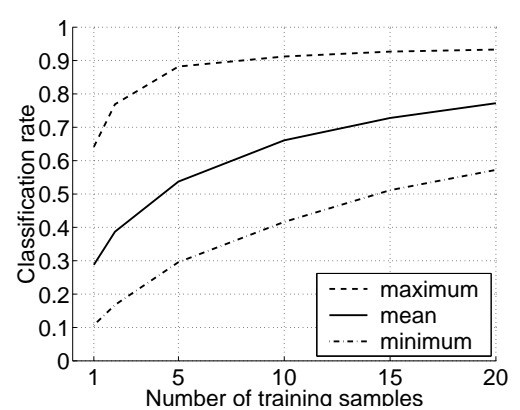

(c) VZ-MRF

Figure 8: Comparative evaluation: Classification rate vs. number of training samples. The higher (dashed) curve shows the maximum classification rate achieved by any of the 25 classes, the middle (solid) curve shows the mean, and the lower (dash-dot) curve shows the minimum. (a) Our method, $(\mathrm{H}+\mathrm{L})(\mathrm{S}+\mathrm{R})$ combination. (b) VZ-Joint method. (c) VZ-MRF method.

Comparative evaluation. The results presented in this section clearly show that our local affine-invariant representation is sufficient for the successful recognition of textures on (possibly non-rigid) surfaces in three dimensions. To answer the question of whether its invariance properties are actually necessary, we have performed a comparative evaluation with the non-invariant algorithm recently proposed by Varma and Zisserman [47]. This method, dubbed VZ in the following, uses a dense set of 2D textons; the descriptors are raw pixel values measured in fixed-size neighborhoods (perhaps surprisingly, this feature representation was found to outperform the more traditional filter banks). We chose the VZ method for comparison because it achieves the best classification rates to date on the CUReT database (up to $97.47 \%$ ). We have tested two variants of VZ: In the first one, texture images are described by one-dimensional texton histograms encoding the joint distribution of all pixel values in the neighborhood; in the second, they are represented using two-dimensional histograms that encode the conditional distribution of the center pixel given its neighborhood. Accordingly, the respective variants are dubbed VZ-joint and VZ-MRF (for Markov Random Field). The details of our implementation are given in the Appendix.

We have compared our method with with VZ-Joint and VZ-MRF using the randomized classification scheme described in Section 4.1. Figure 8 shows the results as a function of training set size. Consistent with the findings of Varma and Zisserman [47], VZ-MRF 
slightly outperforms VZ-Joint (64.75\% vs. $66.09 \%$ for 10 training images), though this is considerably below the $92.61 \%$ rate achieved by our $(\mathrm{H}+\mathrm{L})(\mathrm{S}+\mathrm{R})$ method. A closer look at Figure 8 also confirms the reliance of the VZ methods on multiple prototypes: When the training set size is increased from 10 to 20, the classification rates of VZ-Joint and VZ-MRF improve by over $11 \%$ to $75.89 \%$ and $77.21 \%$, respectively, whereas the performance of our method changes by only $3.42 \%$ (from $92.61 \%$ to $96.03 \%$ ).

The outcome of the comparison validates our intuition that intrinsic representation-level invariance is necessary to achieve robustness to large viewpoint and scale changes, especially in situations when the lack of invariance cannot be fully compensated by storing multiple prototypes of each texture. This is indeed the case for our dataset, which has relatively few sample images per class but high intra-class variability, including non-homogeneous textures and unconstrained non-rigid deformations. By contrast, the CUReT database lacks these sources of variability. In particular, CUReT images have no scale variation (all materials are held at the same distance from the camera, only the orientation is changed), limited in-plane rotation, and the same physical surface patch is represented in all samples. In addition, the appearance of each patch in that database is systematically sampled under different combinations of viewing angles and lighting directions, making it straightforward to select a fixed representative subset of samples for training, as is done in most CUReT evaluations. Our evaluation scheme is a lot more punishing in comparison, as it averages classification rates over many different randomly chosen training sets.

\subsection{Dataset 2: Brodatz Database}

The Brodatz database [4] is perhaps the best known benchmark for texture recognition algorithms. In recent years, it has been criticized because of the lack of intra-class variation that it exhibits. However, we feel that it is premature to dismiss the Brodatz database as a challenging platform for performance analysis. For one, relatively few publications actually report results on the entire database (the only studies known to us are [10, 25, 
$35,50])$. In addition, while near-perfect overall results have been shown for the CUReT database [47], the best (to our knowledge) retrieval performance on the Brodatz database is around $84 \%$ [50]. The reason for this is the impressive diversity of Brodatz textures, some of which are quite perceptually similar, while others are so inhomogeneous that a human observer would arguably be unable to group their samples "correctly". The variety of the scales and geometric patterns of the Brodatz textures, combined with an absence of intraclass transformations, makes them a good platform for testing the discriminative power of an additional local shape channel in a context where affine invariance is not necessary, as described below.

The shape channel. The shape of an affinely adapted region is encoded in its local shape matrix, which can also be thought of as the equation of an ellipse. Let $E_{1}$ and $E_{2}$ be two ellipses in the image plane. We eliminate the translation between $E_{1}$ and $E_{2}$ by aligning their centers, and then compute the dissimilarity between the regions as

$$
\mathrm{d}\left(E_{1}, E_{2}\right)=1-\frac{\text { Area }\left(E_{1} \cap E_{2}\right)}{\text { Area }\left(E_{1} \cup E_{2}\right)} .
$$

In the experiments of this section, we use local shape to obtain two additional channels, HE and LE, corresponding to the ellipses found by the Harris and Laplacian detectors, respectively. Notice that the ellipse ground distance, and consequently all shape-based EMD's, must be between 0 and 1. Because the descriptor-based EMD's lie in the same range, the shape-based EMD's can be combined with them through simple addition.

Finally, it is worth noting that the ellipse "distance" as defined above takes into account the relative orientations of the two ellipses. If it is necessary to achieve rotation invariance, we can simply align the major and minor axes of the two ellipses before comparing their areas.

Results. The Brodatz database consists of 111 images. Following the same procedure as previous evaluations $[25,35,50]$, we form classes by partitioning each image into nine nonoverlapping fragments, for a total of 999 images. Fragment resolution is $215 \times 215$ pixels. 


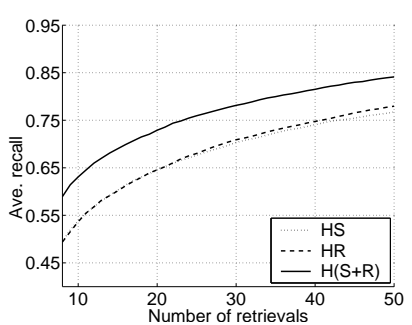

(a)

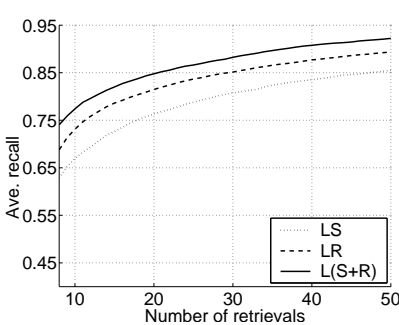

(b)

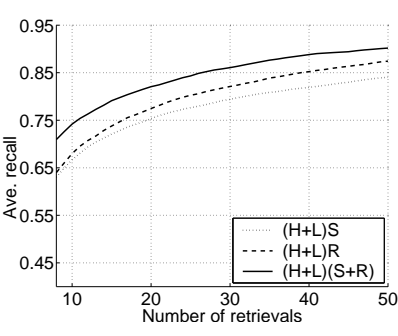

(c)

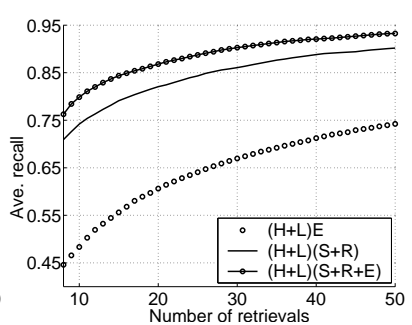

(d)

Figure 9: Retrieval curves for the Brodatz database. (a) Harris descriptor channels. (b) Laplacian descriptor channels. (c) Combined Harris and Laplacian descriptor channels. (d) Comparison of combined performance with and without the ellipse channels (HE and LE).

By comparison with the texture database discussed in the previous section, relatively few regions are extracted from each image: The median values are 132 for the Harris detector, 681 for the Laplacian, 838 combined. Some images contain less than 50 regions total. Given such small numbers, it is difficult to select a fixed number of clusters suitable for all images in the database. To cope with this problem, we replace $k$-means by an agglomerative clustering algorithm that repeatedly merges clusters until the average intra-cluster distance exceeds a specified threshold [17]. This process results in variable-sized signatures, which are successfully handled by the EMD framework. An additional advantage of agglomerative clustering as opposed to $k$-means is that it can be used for the shape channel, since it can take as input the matrix of pairwise distances between the ellipses.

Figure 9 shows retrieval results for the Brodatz database. Similarly to the results of the previous section, the Laplacian channels, shown in part (b) of the figure, have better performance than the Harris channels, shown in part (a). Interestingly, though, for the Brodatz database RIFT descriptors perform better than spin images - the opposite of what we have found in Section 4.2. However, this discrepancy is due at least in part to the variability of signature size (due to the use of agglomerative clustering) in the experimental setup of this section. On average, the RIFT-based signatures of the Brodatz images have more clusters than the spin-based signatures, and we conjecture that this raises the discriminative power of the RIFT channel. Another interesting point is that combining the Harris and Lapla- 
cian channels, as shown in (c), results in a slight drop of performance as compared to the Laplacian channels alone. Finally, (d) shows the effect of adding the shape channels into the mix. By themselves, these channels are relatively weak, since after 8 retrievals the $(\mathrm{H}+\mathrm{L}) \mathrm{E}$ recall is only $44.59 \%$. However, adding these channels to $(\mathrm{H}+\mathrm{L})(\mathrm{S}+\mathrm{R})$ boosts the recall from $70.94 \%$ to $76.26 \%$.

The trends noted above are also apparent in the classification results presented in Table 4. By looking at the first two rows, we can easily confirm the relatively strong performance of the RIFT descriptor (particularly for the Laplacian detector), as well as the marginal drop in performance of the $\mathrm{L}+\mathrm{H}$ channels as compared to $\mathrm{L}$ alone. The latter effect is also seen in the last row of the table, where the $(\mathrm{H}+\mathrm{L})(\mathrm{S}+\mathrm{R}+\mathrm{E})$ classification rate is slightly inferior to the $\mathrm{L}(\mathrm{S}+\mathrm{R}+\mathrm{E})$ rate.

\begin{tabular}{|l|l|l|l|}
\hline & $\mathrm{H}$ & $\mathrm{L}$ & $\mathrm{H}+\mathrm{L}$ \\
\hline $\mathrm{S}$ & 0.6136 & 0.7570 & 0.7531 \\
\hline $\mathrm{R}$ & 0.6000 & 0.8023 & 0.7640 \\
\hline $\mathrm{E}$ & 0.3678 & 0.4932 & 0.5413 \\
\hline $\mathrm{S}+\mathrm{R}+\mathrm{E}$ & 0.7761 & 0.8815 & 0.8744 \\
\hline
\end{tabular}

Table 4: Brodatz database classification results for 3 training samples.

We can get a more detailed look at the performance of our system by examining Figure 10, which shows a histogram of classification rates for all 111 classes using three training samples per class. The histogram reveals that the majority of textures are highly distinguishable, and only a few stragglers are located at the low end of the spectrum. In fact, 36 classes have 100\% classification rate, 49 classes have classification rate at least 99\%, and 73 classes (almost two thirds of the total number of classes) have rate at least $90 \%$. The mean rate is $87.44 \%$. Figure 11 shows four textures that were classified successfully and four textures that were classified unsuccessfully. Not surprisingly, the latter examples are highly non-homogeneous. 


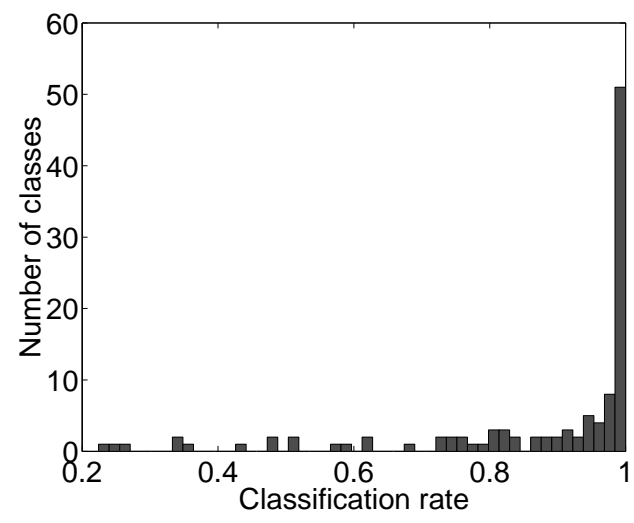

Figure 10: Histogram of classification rates for 3 training samples.

The best retrieval performance curve of our system, corresponding to the $(\mathrm{H}+\mathrm{L})(\mathrm{S}+\mathrm{R}+\mathrm{E})$ combination, has $76.26 \%$ recall after 8 retrievals. This is a slightly higher than the results reported in $[25,35]$, but below $\mathrm{Xu}$ et al. [50], who report $84 \%$ recall using the multiresolution simultaneous autoregressive (MRSAR) model. MRSAR models texture as a stationary random field and uses a dense representation with fixed neighborhood shape and size. A known shortcoming of MRSAR is its limited ability to measure perceptual similarity the method tends to confuse textures that appear very different to human observers [25]. Most significantly, the MRSAR model is difficult to extend with affine invariance. By contrast, our representation is specifically formulated with geometric invariance in mind, is non-parametric, and does not make any statistical assumptions about the input texture.

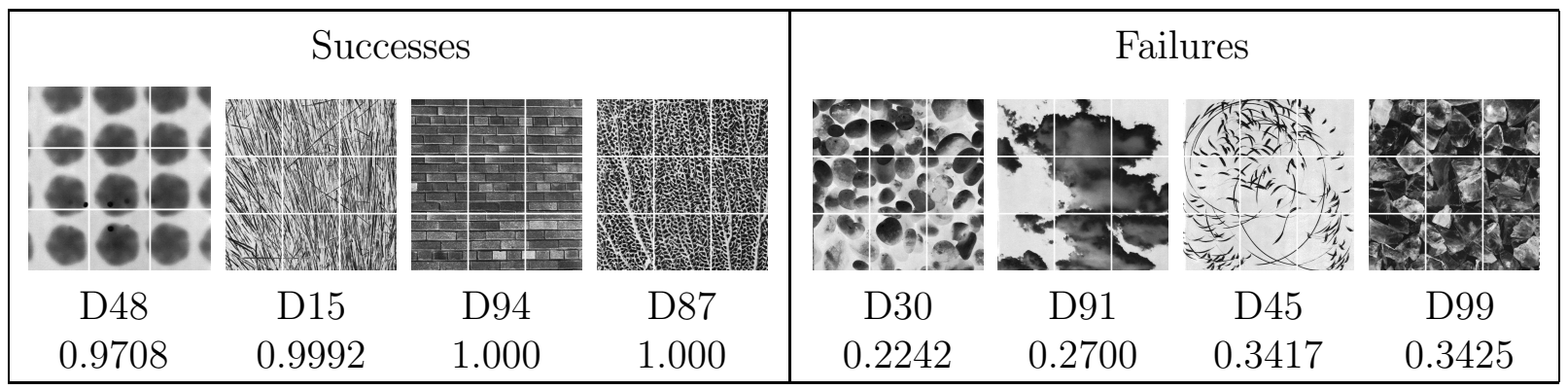

Figure 11: Left: successes. Right: failures. The average classification rates are shown below the corresponding class labels. Note that the representation for D48 has an average number of only 27 combined Laplacian and Harris regions per sample. 


\section{Discussion}

In this article, we have introduced a sparse affine-invariant texture representation that applies spatial and shape selection to automatically determine the locations and support regions of salient local texture regions. In summary, the main contributions of our work are:

- A sparse representation: The experiments of Section 4 show that it is possible to successfully recognize many textures based on information contained in a very small number of image regions.

- Spatial and shape selection: These mechanisms provide robustness against viewpoint changes, non-rigid deformations, and non-homogeneity of the texture pattern. In addition, affine regions capture important perceptual characteristics of many textures.

- Novel intensity-based descriptors: Spin images and RIFT descriptors, presented in Section 3.2, provide a high degree of invariance while serving as a rich description of the intensity pattern of local texture patches.

- A flexible approach to invariance: Our system is flexible in that local shape information may either be discarded or used as a feature, depending on the degree of invariance required by the application.

In our experiments, we have evaluated two detectors and two descriptors on two datasets of about a thousand images each. The first dataset has tested the invariance of our proposed representation to viewpoint changes, as well as complex appearance changes and nonrigid deformations. A comparative evaluation with the VZ method [47] has confirmed that representation-level affine invariance is necessary for achieving good performance on a relatively sparse dataset with high intra-class variability. However, it remains to be determined whether the advantage of our method will persist when the training set can be made to include representative samples of all variations that are likely to appear at testing time. In this case, it is possible that our approach, which throws away potentially useful information 
in the process of computing invariants, would prove less discriminative than an approach based on multiple view-dependent prototypes. We plan to investigate this possibility in future evaluations.

Our second set of experiments, carried out on the Brodatz database, has allowed us to test the descriptive power of the local shape information captured by the affine region detectors. As shown in Figure 9 (d), augmenting our model with an additional shape channel has boosted the recall rate by almost $5 \%$, from $70.94 \%$ to $76.26 \%$. However, because these results are still somewhat inferior to those reported by a non-invariant method [50], one might wonder whether our strategy of factoring out shape in order to compute invariant appearance descriptors, and then adding it back in as a separate "ellipse channel," may actually produce a less expressive model (at least, for this particular database) than an approach that does not separate appearance and shape. Addressing this issue is another interesting direction for further research.

Next, let us briefly summarize the findings of Section 4. The Laplacian detector has shown better overall performance for both texture datasets; nevertheless, as can be seen from Table 3, the Harris detector can have superior performance for certain individual classes. The Harris detector also has the advantage of producing much sparser image representations than the Laplacian. As for descriptors, spin images won over RIFT for the texture database in Section 4.2, while RIFT worked slightly better for the Brodatz database in Section 4.3 (however, recall that the latter comparison is confounded by signature size variability). Despite these differences in performance, it is advantageous to retain both descriptors, as combining their outputs generally improves the accuracy of recognition.

Perhaps the most important high-level observation we can make is that both the descriptors and the detectors tend to fluctuate in performance from texture to texture and from database to database. Two lessons can be drawn from this. On the one hand, researchers working on specific applications of texture analysis should conduct comparative evaluations on representative data to discover which channels or channel combinations would work best 
in their case. On the other hand, to achieve a general understanding of the expressive power of different channels on different types of texture, it is necessary to conduct systematic studies using much larger databases (containing hundreds or even thousands of texture classes), as well as larger numbers of descriptors and detectors, or even parameterized descriptor/detector families. Such studies should focus on quantifying performance as a function of all sources of variability that may be of interest. Currently, our dataset includes some dimensions, like non-rigidity of the surface and inhomogeneity of the texture pattern, that seem difficult to quantify precisely. An important future research direction is the development of systematic evaluation techniques suitable for dealing with large-scale datasets featuring many degrees of freedom.

Another issue requiring further study is the method for combining channels. In our limited evaluation, the simple method of adding the individual EMD matrices has generally proven effective in boosting performance. However, as we have learned from our Brodatz experiments, it is occasionally possible for the combined recognition rate to actually be lower than the single-channel rates. We plan to study more sophisticated methods for combining channels that would not suffer from similar detrimental effects.

Finally, we plan to strengthen the proposed texture representation using spatial relationships between neighboring regions. A few recent representations [28, 42] have used a two-level scheme, with intensity-based textons at the first level and histograms of texton distributions over local neighborhoods at the second level. For many natural textures, the arrangement of affine regions captures perceptually important information about the global geometric structure. Augmenting our representation with such information is likely to increase its ability to distinguish textures that have similar local neighborhoods but different spatial layouts. To date, we have conducted preliminary experiments in classification of individual texture regions using simple co-occurrence relations [20]. We expect that a richer two-level texture representation will be useful for the problem of segmenting and classifying natural images that contain multiple texture categories such as sky, water, plants, and man-made structures. 


\section{Appendix: Implementation of the VZ Method}

At the feature extraction stage, all $N \times N$ pixel neighborhoods in an image are taken and reordered to form $N^{2}$-dimensional feature vectors. In our implementation, $N=11$ for 121-dimensional features. This was selected as the smallest neighborhood size to yield a feature space of higher dimensionality than spin images. To provide some invariance to illumination changes, the vectors are normalized to zero mean and unit norm. To compute the texton dictionary, five images per class are chosen at random, and $20 \%$ of all feature vectors extracted from these images are retained, also at random. This reduction in the number of feature vectors is motivated primarily by the memory limitations of our system. The feature vectors for all 25 classes are clustered using $k$-means into 40 clusters each, resulting in a dictionary of 1000 textons. For the VZ-joint variant, each pixel in an image is labeled by its nearest texton center, and the distribution of all texton labels is represented using a 1000-dimensional histogram. For the VZ-MRF variant, an image is represented using a two-dimensional histogram: For each texton, a conditional distribution of the center pixel is stored as a histogram with 20 bins. This gives us a 20,000-dimensional representation

for each image. In both cases, histograms are compared using the $\chi^{2}$ distance, and nearestneighbor classification is used.

\section{Acknowledgments}

This research was partially supported by the National Science Foundation under grant IIS0308087, the European project LAVA (IST-2001-34405), the UIUC Campus Research Board, the UIUC-CNRS collaboration agreement, and the Beckman Institute for Advanced Science and Technology. We also wish to thank Andrew Zisserman and Manik Varma for many useful discussions, and the anonymous reviewers for their constructive comments that have helped us to improve this article. 


\section{References}

[1] A. Baumberg. Reliable feature matching across widely separated views. In Proc. CVPR, volume 1, pages 774-781, 2000.

[2] S. Belongie, J. Malik, and J. Puzicha. Shape matching and object recognition using shape contexts. IEEE Trans. PAMI, 24(4):509-522, 2002.

[3] D. Blostein and N. Ahuja. A multiscale region detector. Computer Vision, Graphics and Image Processing, 45:22-41, 1989.

[4] P. Brodatz. Textures: A Photographic Album for Artists and Designers. Dover, New York, 1966.

[5] F.S. Cohen, Z. Fan, and M.A.S. Patel. Classification of rotated and scaled textured images using Gaussian Markov field models. IEEE Trans. PAMI, 13(2):192-202, 1991.

[6] J.L Crowley and A.C. Parker. A representation of shape based on peaks and ridges in the difference of low-pass transform. IEEE Trans. PAMI, 6:156-170, 1984.

[7] O.G. Cula and K.J. Dana. Compact representation of bidirectional texture functions. In Proc. CVPR, volume 1, pages 1041-1047, 2001.

[8] K.J. Dana, B. van Ginneken, S.K. Nayar, and J.J. Koenderink. Reflectance and texture of real world surfaces. ACM Transactions on Graphics, 18(1):1-34, 1999.

[9] J. Gårding and T. Lindeberg. Direct computation of shape cues using scale-adapted spatial derivative operators. IJCV, 17(2):163-191, 1996.

[10] B. Georgescu, I. Shimshoni, and P. Meer. Mean shift based clustering in high dimensions: A texture classification example. In Proc. ICCV, pages 456-463, 2003.

[11] R. Haralick. Statistical and structural approaches to texture. Proceedings of the IEEE, 67:786-804, 1979. 
[12] C. Harris and M. Stephens. A combined corner and edge detector. In M. M. Matthews, editor, Proceedings of the 4th Alvey Vision Conference, pages 147-151, 1988.

[13] A. Johnson and M. Hebert. Using spin images for efficient object recognition in cluttered 3d scenes. IEEE Trans. PAMI, 21(5):433-449, 1999.

[14] B. Julesz. Visual pattern discrimination. IRE Transactions on Information Theory, IT-8:84-92, 1962.

[15] B. Julesz. Textons, the elements of texture perception and their interactions. Nature, 290:91-97, 1981.

[16] T. Kadir and M. Brady. Scale, saliency and image description. IJCV, 45(2):83-105, 2001.

[17] L. Kaufman and P. Rousseeuw. Finding Groups in Data: An Introduction to Cluster Analysis. John Wiley \& Sons, New York, 1990.

[18] J. Koenderink and A. Van Doorn. Representation of local geometry in the visual system. Biological Cybernetics, 55:367-375, 1987.

[19] J. Koenderink and A. Van Doorn. The structure of locally orderless images. IJCV, 31(2/3):159-168, 1999.

[20] S. Lazebnik, C. Schmid, and J. Ponce. Affine-invariant local descriptors and neighborhood statistics for texture recognition. In Proc. ICCV, pages 649-655, 2003.

[21] S. Lazebnik, C. Schmid, and J. Ponce. A sparse texture representation using affineinvariant regions. In Proc. CVPR, volume 2, pages 319-324, 2003.

[22] T. Leung and J. Malik. Recognizing surfaces using three-dimensional textons. IJCV, $43(1): 29-44,2001$. 
[23] E. Levina and P. Bickel. The Earth Mover's distance is the Mallows distance: Some insights from statistics. In Proc. ICCV, volume 2, pages 251-256, 2001.

[24] T. Lindeberg. Feature detection with automatic scale selection. IJCV, 30(2):77-116, 1998.

[25] F. Liu and R. W. Picard. Periodicity, directionality, and randomness: Wold features for image modeling and retrieval. IEEE Trans. PAMI, 18(7):722-733, 1996.

[26] X. Llado, J. Marti, and M. Petrou. Classification of textures seen from different distances and under varying illumination direction. In IEEE Int. Conf. Image Processing, volume 1, pages 833-836, 2003.

[27] D. Lowe. Distinctive image features from scale-invariant keypoints. IJCV, 60(2):91-110, 2004.

[28] J. Malik, S. Belongie, T. Leung, and J. Shi. Contour and texture analysis for image segmentation. IJCV, 43(1):7-27, 2001.

[29] J. Malik and P. Perona. Preattentive texture discrimination with early vision mechanisms. J. Opt. Soc. Am. A, 7(5):923-932, 1990.

[30] J. Mao and A. Jain. Texture classification and segmentation using multiresolution simultaneous autoregressive models. Pattern Recognition, 25:173-188, 1992.

[31] J. Matas, O. Chum, U. Martin, and T Pajdla. Robust wide baseline stereo from maximally stable extremal regions. In Proc. BMVC, volume 1, pages 384-393, 2002.

[32] K. Mikolajczyk and C. Schmid. Indexing based on scale invariant interest points. In Proc. ICCV, pages 525-531, 2001.

[33] K. Mikolajczyk and C. Schmid. An affine invariant interest point detector. In Proc. ECCV, volume 1, pages 128-142, 2002. 
[34] K. Mikolajczyk and C. Schmid. A performance evaluation of local descriptors. In Proc. CVPR, volume 2, pages 257-263, 2003.

[35] R. Picard, T. Kabir, and F. Liu. Real-time recognition with the entire Brodatz texture database. In Proc. CVPR, pages 638-639, 1993.

[36] J. Puzicha, Y. Rubner, C. Tomasi, and J. Buhmann. Empirical evaluation of dissimilarity measures for color and texture. In Proc. ICCV, volume 2, pages 1165-1172, 1999.

[37] T. Randen and J. Husøy. Filtering for texture classification: A comparative study. IEEE Trans. PAMI, 21(4):291-310, 1999.

[38] Y. Rubner and C. Tomasi. Texture-based image retrieval without segmentation. In Proc. ICCV, pages 1018-1024, 1999.

[39] Y. Rubner, C. Tomasi, and L. Guibas. The Earth Mover's distance as a metric for image retrieval. IJCV , 40(2):99-121, 2000.

[40] F. Schaffalitzky and A. Zisserman. Viewpoint invariant texture matching and wide baseline stereo. In Proc. ICCV, volume 2, pages 636-643, 2001.

[41] F. Schaffalitzky and A. Zisserman. Multi-view matching for unordered image sets, or "How do I organize my holiday snaps?". In Proc. ECCV, volume 1, pages 414-431, 2002.

[42] C. Schmid. Constructing models for content-based image retrieval. In Proc. CVPR, volume 2, pages 39-45, 2001.

[43] C. Schmid and R. Mohr. Local greyvalue invariants for image retrieval. IEEE Trans. PAMI, 19(5):530-535, 1997. 
[44] J. Sivic and A. Zisserman. Video google: A text retrieval approach to object matching in videos. In Proc. ICCV, pages 1470-1477, 2003.

[45] T. Tuytelaars and L. Van Gool. Matching widely separated views based on affinely invariant neighbourhoods. IJCV, 59(1):61-85, 2004.

[46] M. Varma and A. Zisserman. Classifying images of materials: Achieving viewpoint and illumination independence. In Proc. ECCV, volume 3, pages 255-271, 2002.

[47] M. Varma and A. Zisserman. Texture classification: Are filter banks necessary? In Proc. CVPR, volume 2, pages 691-698, 2003.

[48] H. Voorhees and T. Poggio. Detecting textons and texture boundaries in natural images. In Proc. ICC , pages 250-258, 1987.

[49] J. Wu and M. J. Chantler. Combining gradient and albedo data for rotation invariant classification of 3d surface texture. In Proc. ICCV, volume 2, pages 848-855, 2003.

[50] K. Xu, B. Georgescu, D. Comaniciu, and P. Meer. Performance analysis in content-based retrieval with textures. In Proc. Int. Conf. Patt. Recog., volume 4, pages 275-278, 2000.

[51] J. Zhang, P. Fieguth, and D. Wang. Random field models. In A. Bovik, editor, Handbook of Image and Video Processing, pages 301-312. Academic Press, San Diego, CA, 2000. 